\title{
Stochastic optics: A local realistic analysis of optical tests of Bell inequalities
}

\author{
Trevor W. Marshall \\ Department of Mathematics, University of Manchester, Manchester M13 9PL, United Kingdom \\ Emilio Santos \\ Departamento de Física Moderna, Universidad de Cantabria, Santander, Spain \\ (Received 2 May 1988; revised manuscript received 22 November 1988)
}

\begin{abstract}
Stochastic optics may be considered as simply a local realistic interpretation of quantum optics and, in this sense, it is a first step in the reinterpretation of the whole of quantum theory. However, as it is not possible to interpret all the details of quantum theory in a local realistic manner, as shown by Bell's theorem, minor changes are introduced in the formalism with the consequence that the new theory makes different predictions in some special cases. In stochastic optics, the quantum-operator formalism is simply considered a formal way of dealing with stochastic fields. In particular, the quantum zero point is taken as a real random electromagnetic radiation filling the whole of space. This radiation noise has the same nature as light signals, the only difference being the greater intensity of the latter. We assume that photon detectors have an intensity threshold just above the level of the noise, thus detecting only signals. Transmission of radiation through polarizers follows Malus's law, but the interplay of signal and noise leads quite naturally to the prediction that the detection probability of some signals is enhanced, which is known to be a necessary condition for the violation of the empirically tested Bell inequalities. In our view, correlated photon pairs are pairs of light signals supercorrelated in polarization, in the sense that, as well as the signal, the accompanying noise is also correlated. Thus stochastic optics allows predictions for the empirical correlations very close, but not identical, to the quantum ones. The theory is applied to the analysis of all experiments designed to test the Bell inequalities by measuring polarization correlations of photon pairs. The predictions agree with quantum optics and experiments within statistical errors, except for the Holt-Pipkin experiment. In this case, the experimental results agree with stochastic optical predictions within two standard deviations while violating quantum optics by four.
\end{abstract}

\section{INTRODUCTION}

More than 80 years have elapsed since Einstein introduced the concept of a "photon", in order to explain the discreteness of the absorption of light by material systems. Wave-particle dualism, an essential part of the photon concept, has since been extended to the whole of quantum theory, giving rise to conceptual difficulties that nonbody has been able to clarify in a completely satisfactory manner. In spite of these difficulties it is now widely believed that no alternative exists to the present paradigm; that is, nature cannot be interpreted along classical lines. In the domain of optics, a number of experiments have been performed in the last two decades to show nonclassical effects in the statistical properties of light. However, the claim that these experiments cannot be interpreted classically is incorrect. In fact, we have developed a pure wave theory of light, able to interpret all these experiments, at least qualitatively. The theory, which we call stochastic optics, has been presented in a recent paper, ${ }^{1}$ where we have shown in particular how the theory explains (i) the apparent corpuscular behavior of light (ii) the results of optical tests of Bell's inequalities, and (iii) photon antibunching. In the present paper we analyze in more detail the second class of experiments.

The optical tests of the Bell inequalities, ${ }^{2-8}$ by measuring "photon" polarization correlations, are the most re- markable of all the experiments designed to show the nonclassical behavior of nature. In fact, Bell's theorem shows that there are some specific predictions of quantum mechanics which are incompatible with any local realistic model. Then it is, in principle, possible to test quantum mechanics against the whole class of realistic local theories. In practice, however, it is rather difficult to perform crucial tests and, until now, only atomic cascade experiments (Sec. IV) are considered reliable. The performed experiments have given results in agreement with the quantum predictions, with one exception (see Sec. IV). As a consequence, a large fraction of the scientific community now believes that local realistic theories have been refuted empirically. The main purpose of the present paper is to show that this opinion is incorrect. We shall show that stochastic optics, a realistic and local theory, offers a natural explanation of the results obtained in these experiments. Furthermore, it explains also the results of the one experiment that did not agree with the quantum prediction, thus offering for the first time an explanation of that anomaly.

Stochastic optics is a purely classical theory of light derived from Maxwell's electromagnetic theory. Thus it is a "wave" or "field" theory of light. The only difference between it and conventional classical optics is the assumption that there is a universal noise or random background radiation filling the whole of space. This noise is 
just the zero-point field of quantum electrodynamics, but taken as real instead of "virtual" (not a well-defined concept in any case). The idea that the zero-point field may explain the peculiarities of the quantum behavior goes back to Planck and Nernst at the beginning of the century, but the systematic study of classical systems of charges moving under the action of the zero-point field (known as stochastic electrodynamics, see for reviews Refs. 9-11) is only 30 years old. Stochastic optics has a more narrow scope than stochastic electrodynamics, as it does not attempt a detailed study of the interaction of light with matter but considers only the interaction with macroscopic bodies, such as lenses, mirrors, polarizers, etc. We assume for these interactions the same laws as in classical optics, but including the zero-point radiation. For the emission and absorption of light it is necessary to make phenomenological assumptions, as explained below. We emphasize that stochastic optics is still at a preliminary stage of development and does not yet allow accurate predictions to be tested against quantum mechanics. However, the theory gives a simple explanation of the most dramatic quantum aspects of optics. With a suitable choice of just two adjustable parameters, it is possible to fit all performed experiments within statistical errors. It must be noted that we shall consider only radiation able to interact collectively with macroscopic devices, thus excluding radiation with wavelengths shorter than the near ultraviolet. As the zero-point radiation is irrelevant for wavelengths larger than those in near infrared, the specific range of our interest corresponds therefore to the traditional domain of optics.

In the following, we summarize the principles of stochastic optics, referring for details to our previous paper. ${ }^{1}$ The essential hypothesis is that there is everywhere in space a zero-point noise which cannot be directly detected, because only radiation "above the sea" of zero point can activate light detectors. In most cases, optical devices, such as lenses, mirrors, etc., transmit radiation in such a way that the part below (above) the "sea level" remains below (above), i.e., as noise (signal). In consequence, the laws of classical optics apply. We have defined these devices to belong to class I. There are class-II devices, such as partially transparent mirrors or polarizers, which divide the incoming radiation in two parts going to different channels. In this case, there is also some amount of noise which is similarly divided and is able to mix with the two parts of the signal giving either constructive or destructive interference. Our proposal is that quantum behavior in the transmission of light is due to this interference. For instance, a signal arriving at a semitransparent mirror gives, by the interference with the noise, two outgoing signals, but only one of them with enough intensity (above the sea level) to be detected. This explains the "particle" behavior of light ("the photon goes in only one channel"). However, in the other channel there is radiation "below the sea," carrying information of the incoming signal, so that a recombination of the beams in the two channels may give rise to interference fringes showing that light is a wave.

In the present paper, we shall follow a more formal approach than in the previous one, ${ }^{1}$ in order to show the closeness of stochastic and quantum optics. Indeed, stochastic optics could be considered as simply a local realistic interpretation of quantum optics. In this sense, it is a first step (confined to the domain of optics) in the reinterpretation of the whole of quantum theory. However, it is not possible to interpret all the details of quantum theory in a local realistic manner (as shown by Bell's theorem ${ }^{2,3}$ ) without some change in the formalism. In consequence, stochastic optics cannot be simply a reinterpretation of quantum optics but a new theory making different predictions in some special cases. Therefore, the two theories may be distinguished empirically, as we shall show later. In contrast with our previous paper, ${ }^{1}$ where we described stochastic optics as a natural modification of standard classical optics once the existence of a universal noise is accepted, we shall start here from the quantum formalism. We shall argue that stochastic optics is the "minimal" modification of quantum optics needed to interpret the theory classically (i.e., as realistic and local).

\section{PRINCIPLES OF STOCHASTIC OPTICS}

For our purposes it is convenient to consider light signals with a definite polarization and phase. One such signal is classically represented by the electric field (similarly for the magnetic field) in the form

$$
\mathbf{E}(\mathbf{r}, t)=\operatorname{Re}\left[f(\mathbf{r}, t) e^{i \chi} \mathbf{V}\right], \quad \mathbf{V}=\left(E \cos \phi, E(\sin \phi) e^{i \Psi}\right),
$$

where $\phi$ and $\Psi$ are polarization parameters and $\chi$ the phase, with the domains

$$
0 \leq \phi \leq \pi / 2, \quad 0 \leq \Psi, \chi \leq 2 \pi,
$$

and the function $f(\mathbf{r}, t)$ contains the space-time dependence. Then the parameter $E$ represents the amplitude of the signal, and the intensity (total energy) is given, in suitable units, by $E^{2}$.

The quantum description of radiation is given in terms of vectors and operators in Hilbert space. ${ }^{12}$ The electric and magnetic fields become operators and the state of the field (containing, e.g., a light signal) is given by a vector $|\alpha\rangle$. The most relevant observable is the energy, and the corresponding operator, the Hamiltonian, is written

$$
H=\sum_{j} h v_{j} a_{j}^{\dagger} a_{j},
$$

where $a^{\dagger}(a)$ is the creation (annihilation) operator of one "photon." It is possible to consider many kinds of light (thermal, coherent, etc.) but in the present paper we will study only "one-photon" signals. This is because all nonclassical behavior has been shown with this kind of light. The average and mean-square energies of that state are

$$
\varepsilon=\langle 1|H| 1\rangle=h v, \quad \varepsilon^{2}=\left\langle 1\left|H^{2}\right| 1\right\rangle=h^{2} v^{2},
$$

thus showing that the value $h v$ of the energy is sharp (not fluctuating).

Stochastic optics attempts to interpret quantum optics with a realistic philosophy. Then, we must know whether the zero-point radiation really exists. The orthodox interpretation does not answer this question and hides the 
problem with the statement that the zero-point radiation is "virtual." Until now, attempts to find a classical or semiclassical alternative to quantum optics have not taken into account the zero-point radiation and have failed. A typical example was the semiclassical theory of Jaynes. ${ }^{13}$ We assume that the zero point is a real radiation. This implies the first modification of the quantum formalism consisting in replacing the Hamiltonian (2.3) by

$$
H=\sum_{j} \frac{1}{2} h v_{j}\left(a_{j}^{\dagger} a_{j}+a_{j} a_{j}^{\dagger}\right)=\sum_{j} h v_{j}\left(a_{j}^{\dagger} a_{j}+\frac{1}{2}\right) .
$$

This is a rather natural change because (2.5) is what we obtain from a straightforward quantization of the classical energy, while the operator reordering (or, equivalently, the removal of the term $\frac{1}{2} h v$ ) is a rather ad hoc procedure for the elimination of the undesirable infinite energy of the vacuum. With our modification, the average energy of the radiation mode associated with the photon is no longer $h v$ but $\frac{3}{2} h v$. On the other hand, every vacuum mode has an average energy $\frac{1}{2} h v$.

For reasons to be explained at the end of this section, we will not follow the standard treatment, where each radiation mode is considered to have a definite wave vector [or, more generally, space-time function $f$, see (2.1)] and polarization. We shall instead superpose the two modes having the same wave vector to get one component of the electric field with a given elliptical polarization [described by (2.1)]. Then, if the pair of modes contains a onephoton signal, its energy will be $2 h v$; if it corresponds to the vacuum, its energy will be $h v$. Each pair of modes can be associated with one cell of volume $8 \pi^{3}$ in sixdimensional space (i.e., the space of three coordinates and three wave-vector components). Therefore, we shall analyze the full radiation field in any region of space as a superposition of field components, each corresponding to one cell and having a well-defined elliptical polarization.

In any particular case, once we define the radiation modes appropriate for the problem at hand, the full radiation field can be described by giving four parameters, $E$, $\phi, \Psi$, and $\chi$, for each cell [see (2.1)]. (In the normal treatment there are two parameters, amplitude and phase, for each mode, which gives again four per cell.) As appropriate to a stochastic theory, the four parameters are random variables. From symmetry considerations it is not difficult to find the probability distribution for the three angular parameters (see below), but it is not so easy to find the probability distribution of the amplitude parameter $E$. We know the average value of its square (namely, $h v$ for a vacuum cell and $2 h v$ for a cell containing one "photon") but we need all the moments in order to know the full probability distribution. As we want to remain as close as possible to the quantum formalism, these moments should be computed using the Hamiltonian (2.5) and the standard quantum rules. What we obtain is that the energy of each mode is dispersion free. Apparently, this solves our problems and suggests putting the following probability density for the parameters of a vacuum cell:

$$
\rho\left(E_{0}, \phi_{0}, \Psi_{0}, \chi_{0}\right)=\left(4 \pi^{2}\right)^{-1} \sin \left(2 \phi_{0}\right) \delta\left(E_{0}-1\right),
$$

where $E_{0}^{2}$ is given in units $h v$.

As said above, the distribution for the angular parameters has been obtained by symmetry considerations. ${ }^{14}$ Not only is it rotationally invariant, but it can be shown to remain unchanged when a beam crosses a $\lambda / n$ plate, for any $n$, so that (2.6) is quite satisfactory in this respect. However, the sharp value of the amplitude $E_{0}$ cannot be physical. In fact, there are strong arguments ${ }^{15}$ for a Gaussian distribution of amplitudes, and certainly (2.6) is not obtained in general for one cell by a superposition of two radiation modes with arbitrary phases. ${ }^{16}$ Consequently, further changes in the quantum formalism will be required, besides substituting (2.5) for (2.3), but we leave this problem for future work and, for the moment, we shall use (2.6) unamended.

Now, we must specify the distribution of polarizations of the signals coming from an atomic source. Obviously this will depend on the characteristics of the source, but in the present paper we will consider only sources with rotational invariance. In this case, we propose for the ensemble of signals the maximum possible symmetry that is a distribution similar to (2.6)

$$
\rho(E, \phi, \Psi, \chi)=\left(4 \pi^{2}\right)^{-1} \sin (2 \phi) \delta(E-\beta) .
$$

As this corresponds to the field component of one cell (containing two radiation modes) the energy should be $2 h v$, and therefore we should take $\beta^{2}=2$ in (2.7). Howev$\mathrm{er}$, as we are using a somewhat nonphysical distribution (namely, with sharp energy), we propose to compensate for this fact by treating $\beta$ as an adjustable parameter (the value $\beta^{2}=2.2$ will be obtained in Sec. III as the optimum choice). It is not necessary to specify the form of the space-time function $f(\mathbf{r}, t)$ [see (2.1)] but it seems likely that the region where it is not zero corresponds to what Einstein called Nadelstrahlung (needle radiation), i.e., a region of the order of meters in the direction of propagation and a few wavelengths in diameter. A wave packet of this form and size (superimposed on the zero-point field) is what plays for us the role of one "photon."

Stochastic optics has a limited scope because it is confined to the transmission of radiation (including zeropoint radiation) through macroscopic devices. So, it does not cover the emission or absorption of light signals or, in general, the microscopic interaction of radiation with matter. In this respect it is similar to semiclassical theories. However, we want a fully classical theory able to be interpreted in a local realistic way. Consequently, we do not use the quantum theory of absorption and emission, but replace it by simple phenomenological assumptions. These assumptions, however, will stay as close as possible to the quantum formalism.

In quantum optics, the detection probability of a signal is usually given by the expectation value of the number operator multiplied by the efficiency factor $\eta$ (for simplicity we ignore the dependence of $\eta$ on frequency), that is,

$$
P=\eta\langle\alpha|N| \alpha\rangle, \quad N=\sum_{j} a_{j}^{\dagger} a_{j}
$$

Now, the number operator $N$, like the Hamiltonian (2.3), has the operators $a$ and $a^{\dagger}$ in the "normal ordering," that is, $a$ to the right. This is equivalent to subtracting the 
noise, i.e., assuming that zero-point radiation does not activate photodetectors. To bring our description close to quantum theory, the same assumption is made in stochastic optics and we shall write the detection probability as

$$
P=\eta \sum_{j}\left(I_{j}-1\right),
$$

where $I_{j}$ is now the total energy in one cell of sixdimensional space (two radiation modes) in units $h v$. We shall call "ideal detector" any detector fulfilling our (2.9), which is equivalent to the quantum rule (2.8). Ideal detectors, however, are impossible by the very nature of stochastic optics, as explained below. Therefore, some difference with quantum optics can be predicted in experiments where the nonideal behavior of detectors is important.

The reason that ideal detectors are impossible within stochastic optics is that the zero-point radiation is random, so that the "sea level" cannot be sharply defined, as was pointed out earlier. In other words, fluctuations of the noise can always be confused with signals. In a more formal way the difficulty can be seen in Eq. (2.9). In fact, if fluctuations exist, the intensity $I$ can be smaller than the average of the noise, thus making the probability $P$ negative, which is absurd. The problem is that we must find an alternative to (2.8) fulfilling the two conditions of being non-negative and giving a detection probability proportional to the energy above the zero-point sea. But these two conditions are clearly incompatible if there are fluctuations in the zero-point field. At least two predictions seem unavoidable in stochastic optics: (i) there will be always some dark rate in any detector (a well-known fact in real experiments) and (ii) the efficiency of detectors should remain low if we want to avoid a high dark rate. For the moment, the simplest solution seems to be to replace (2.9) by a detection probability which is a function of the signal intensity with a threshold. So we shall assume for the probability, instead of (2.9)

$$
P=\xi \sum_{j}\left\langle\left(I_{j}-\gamma\right)_{+}\right\rangle, \quad \eta=\xi\left(\beta^{2}-\gamma\right),
$$

where $\gamma$ is some threshold energy (in units $h v$ ) and $\eta$ is the "photon" efficiency. The notation $(\cdots)_{+}$means putting zero if the argument is negative and $\langle\cdots\rangle$ means the ensemble average over the noise with the distribution (2.6). Equation (2.10) is more physical than (2.9) because it does not allow negative probabilities, even if the noise intensity fluctuates. It must be taken into account that $I_{j}$ contains the energy of the signal both above and below the sea of zero-point noise, and that the part below the sea has a unit $(h v)$ of energy. Then $\gamma$ must be greater than unity in order to detect no noise, but close to it in order to detect most of the signals.

However, this clearly shows the two differences with quantum optics already noted. Either weak signals are not detected (if $\gamma \gg 1$ ) or there will be a lot of noise detected in the form of dark background (if $\gamma$ is close to 1). The difficulty can be diminished in practice by using low-efficiency detectors. We think that this is a real problem for actual laboratory practice, which stochastic optics explains better than quantum optics. Further- more, our parameter $\gamma$ corresponds to the adjustable voltage bias in actual photomultipliers. Therefore, the existence of this adjustable parameter is rather a merit than a shortcoming of stochastic optics. Unlike quantum optics, which gives predictions only for "ideal" measurements, stochastic optics is more physical and predicts, quite naturally, nonideal behavior.

We stress the distinction between the general principles of stochastic optics (propagation of light according to classical laws and existence of zero-point noise) from the simplified model for the noise, the emission, and the absorption, given by Eqs. (2.6), (2.7), and (2.10). This model is provisional and should be improved in the future, while retaining its qualitative differences with quantum optics. Consequently, the predictions of stochastic optics are, for the moment, only semiquantitative.

\section{ACTION OF A POLARIZER}

Our aim in this section is to describe the transformation of a "single-photon" signal when it crosses a real polarizer. To do that, we first consider the action of an ideal polarizer on a monochromatic light beam according to classical optics. Then, we study the single-photon signal crossing an ideal polarizer in stochastic optics, i.e., including the zero-point noise. Finally, we consider the case of real polarizers, especially piles of plates and calcites.

A monochromatic wave with general (elliptical) polarization can be represented by the two Cartesian components of the electric field $\left(E_{x}, E_{y}\right)$, as in (2.1). A twochannel perfect polarizer that transmits the $x$ component and reflects the $y$ component gives the two beams

$$
\left(E_{x}, 0\right) \text { (transmitted), }\left(0, E_{y}\right) \text { (reflected), }
$$

In order to study the behavior of the ideal two-channel polarizer according to quantum optics, we must consider the radiation modes involved. For a given wave vector coming from the left there are two modes with perpendicular linear polarizations. We shall label by $a_{x}^{\dagger}$ and $a_{y}^{\dagger}\left(a_{x}\right.$ and $a_{y}$ ) the creation (annihilation) operators associated to these modes. One of the modes is transmitted and the other one reflected in the polarizer, as shown in Fig. 1, left (solid line). However, in the outgoing transmitted channel there must be another mode with the same wave vector, but perpendicular polarization (represented by a dashed line in the figure), whose associated creation (annihilation) operator we label $b_{y}^{\dagger}\left(b_{y}\right)$. This can only come from the radiation mode entering from above, as shown in the figure. In practice, light signals enter only on one side of the polarizer, say, the left, so that the modes labeled with $b$ are not excited and they can usually be ignored.

In stochastic optics everything is similar, except that the field operators $E_{x}, E_{y}, E_{0 x}$, and $E_{0 y}$ (see Fig. 1, right) become random variables with probability distributions that can be obtained from our assumptions in Sec. II and the laws of propagation of the different radiation modes. Now, although we may ignore the zero-point radiation in all other vacuum modes, it is not possible to ignore the field in the modes labeled $b$. Then, the relevant part of 
the electric field in the transmitted and reflected beams will have electric field vectors of the forms $\left(E_{x}, E_{0 y}\right)$ and $\left(E_{0 x}, E_{y}\right)$, respectively, where the "relevant part" of the noise is

$\left(E_{0 x}, E_{0 y}\right)=\left(E_{0} \cos \phi_{0} \exp \left(i \chi_{0}\right), E_{0} \sin \phi_{0} \exp \left(i \Psi_{0}+i \chi_{0}\right)\right)$.

We now consider that the beam emerging from the first channel of a polarizer crosses another perfect polarizer at an angle $\theta^{\prime}$ with the first one. Then, the signals transmitted through that polarizer have a component parallel to the polarizer axis and another component (coming from a new intervention of the noise) perpendicular. The outgoing signal is represented by
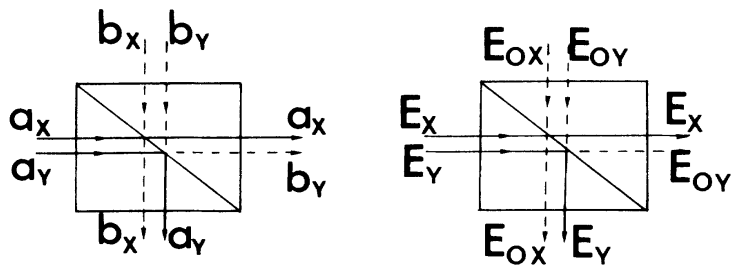

FIG. 1. The action of an ideal two-channel linear polarizer in quantum and stochastic optics. The incoming signal $\mathbf{E}$ is mixed with the zero-point component $\mathbf{E}_{0}$ and split to produce two signals whose total intensity is $E^{2}+E_{0}^{2}$. The axis of the polarizer is taken to be in the $x$ direction.

$$
\left[\beta \cos \phi \cos \theta^{\prime} \exp (i \chi)+\sin \phi_{0} \sin \theta^{\prime} \exp \left(i \Psi_{0}+i \chi_{0}\right), \sin \phi_{1} \exp \left(i \Psi_{1}+i \chi_{1}\right)\right]
$$

Now, we shall consider perfect polarizers combined with real detectors [fulfilling (2.10)]. We must begin by calculating the average intensity of the signals in the transmitted beam when the incoming signals all have the same elliptical polarization. For generality we consider now a polarizer at angle $\theta$, so that the transmitted part of the signal parallel to the polarizer axis is

$$
E_{x}=\beta\left(\cos \phi \cos \theta+\sin \phi \sin \theta e^{i \Psi}\right) e^{i \chi},
$$

while the noise gives a contribution perpendicular to the axis

$E_{0 y}=\left[\cos \phi_{0} \sin \theta+\sin \phi_{0} \cos \theta \exp \left(i \Psi_{0}\right)\right] \exp \left(i \chi_{0}\right)$.

The intensity is obtained adding the square moduli of (3.4) and (3.5):

$$
I=\beta^{2} \cos ^{2} \Phi+\sin ^{2} \Phi_{0},
$$

where we have defined

$\cos (2 \Phi)=\cos (2 \phi) \cos (2 \theta)+\sin (2 \phi) \sin (2 \theta) \cos \Psi$,

and a similar expression for $\Phi_{0}$. Now, the detection probability of a signal will be, according to (2.10)

$$
P(\Phi)=\xi\left\langle\left(\beta^{2} \cos ^{2} \Phi+\sin ^{2} \Phi_{0}-\gamma\right)_{+}\right\rangle_{\Phi_{0}},
$$

an expression that we will frequently use in the future. The average in (3.8) should be made for $\phi_{0}$, and $\Psi_{0}$ with the distribution (2.6) or, equivalently, ${ }^{16}$ for $\Phi_{0}$ with the weighting factor $\sin \left(2 \Phi_{0}\right)$.

The function $P(\Phi)$, represented in Fig. 2, is not very complex but, for later convenience, we shall derive a simple approximation of it by retaining the first two terms in an appropriate series expansion. As the distribution of $\Phi$ contains the weighting factor $\sin (2 \Phi)$, the most suitable expansion is in terms of Legendre polynomials of the variable $\cos (2 \Phi)$. Introducing the ratio $Q(\Phi)$ between the probability with and without polarizer we have

$$
Q(\Phi)=P(\Phi) /\left[\xi\left(\beta^{2}-\gamma\right)\right]=\sum_{n} a_{n} P_{n}(\cos 2 \Phi),
$$

where $P_{n}(x)$ is the $n$th Legendre polynomial with the usual normalization [i.e., $P_{n}(1)=1$ ].

After that, we are in a position to determine the values of the adjustable parameters $\beta$ and $\gamma$. As we will discuss at the end of Sec. IV, exact agreement with quantum optics in the ideal experiments would be obtained with the choice

$$
[Q(\Phi)]_{q}=\frac{1}{2}+(\sqrt{3} / 2) \cos (2 \Phi),
$$

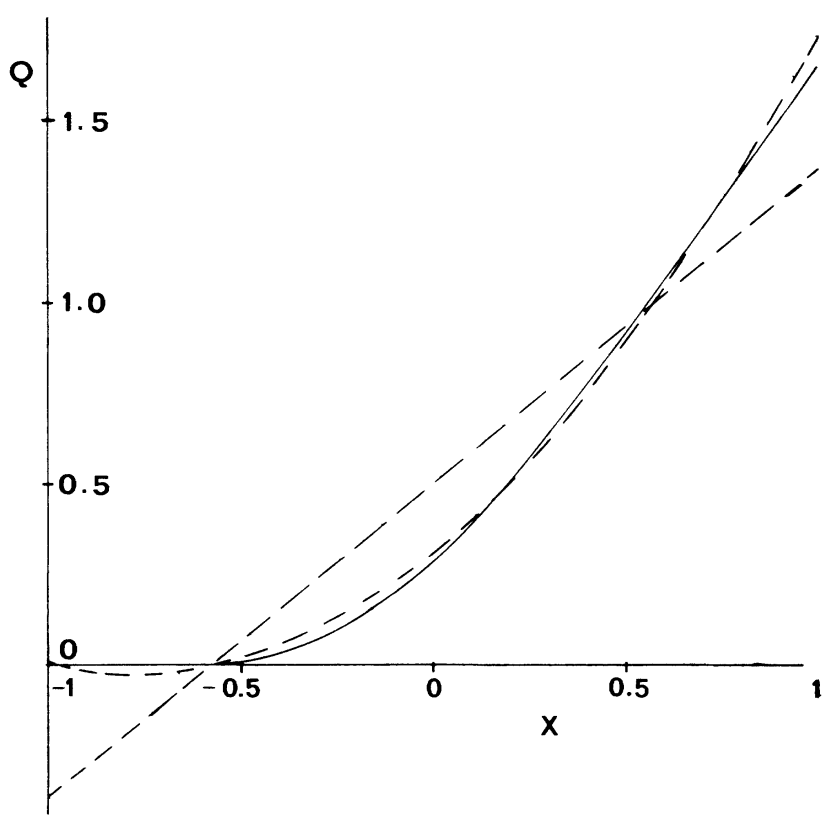

FIG. 2. The function $Q(x)$, representing the ratio of detection probabilities with and without a polarizer set at $\theta=0$, of a "one-photon" light signal having elliptical polarization parameters $(\phi, \Psi, \chi)$, where $x=\cos (2 \Phi)$. The function itself is the solid curve, the two dashed curves giving the approximation by two and three Legendre polynomials, respectively. The first of these-the straight line-represents what we consider to be the quantum approximation to stochastic optics. 
where the subscript denotes quantum. However, this expression can be negative, which is absurd for a ratio of probabilities, in sharp contrast with the more physical expression (3.8). This is another example where quantum optics seems to be just a mathematical approximation of a more physical theory, which we are searching for. Accordingly, we choose $\beta$ and $\gamma$ in accordance with our general practice of remaining as close as possible to quan- tum theory. Then, we fix the first two Legendre polynomials in (3.9) to be $a_{0}=\frac{1}{2}, a_{1}=\sqrt{3} / 2$, which leads to

$$
\beta^{2}=2.204, \quad \gamma=\frac{1}{2}\left[\beta^{2}+1-\left(\beta^{4}-2 \beta^{2}-\frac{1}{3}\right)^{1 / 2}\right]=1.431
$$

After that, (3.9) becomes [writing $x$ for $\cos (2 \Phi)$ ]

$$
Q(\Phi)=\frac{1}{2}+(\sqrt{3} / 2) x+0.3716\left(3 x^{2}-1\right) / 2-0.0568\left(5 x^{3}-3 x\right) / 2-0.0534\left(35 x^{4}-30 x^{2}+3\right) / 8+\cdots
$$

Figure 2 shows that the approximation with two terms (the "quantum limit") is not too bad, and it is very good with three. The choice $a_{0}=\frac{1}{2}$ corresponds to demanding that, when an ensemble of signals from a rotationally invariant source [see (2.7)] crosses a perfect polarizer, the counting rate is just half the rate without the polarizer. This gives the relation between $\beta$ and $\gamma$ shown in (3.11).

An interesting question is whether Malus's law is fulfilled with ideal polarizers but real detectors [behaving as in (2.10)]. The calculation is straightforward. It is enough to consider the change of a signal emerging from the first polarizer when it crosses another (perfect) polarizer at an angle $\theta^{\prime}$ with respect to the first one. The components, parallel and perpendicular respectively, to the second polarizer's axis are

$$
E_{\|}=E_{x} \cos \theta^{\prime}+E_{0 y} \sin \theta^{\prime}, \quad\left|E_{1}\right|=\sin \Phi_{1},
$$

where $E_{x}$ and $E_{0 y}$ are given by (3.4) and (3.5), and $\Phi_{1}$ corresponds to the polarization parameters of the relevant noise intervening at the second polarizer. The ratio between the counting rates with two polarizers and with only one is given by

$$
R\left(\theta^{\prime}\right)=\xi\left\langle\left(\left|E_{\|}\right|^{2}+\left|E_{1}\right|^{2}-\gamma\right)_{+}\right\rangle /\langle P(\Phi)\rangle,
$$

where $E_{\|}$and $E_{\perp}$ given by (3.13), $P(\Phi)$ by (3.8), and the averages are taken over all polarization parameters of signal and noise. A numerical calculation has been made and the results are given in Table I, where they are compared with those predicted by Malus's law. Note that we may exploit the isotropy of the distributions (2.6) and (2.7) and do the calculation for the special case $\theta=0$, since only the relative orientation of the two polarizers is important. In that case (3.13) reduces to (3.3). We see that the agreement is good although not perfect.

By comparison, we include in Table $I$ the results ob- tained with Eq. (3.14) using other values of $\beta$ and $\gamma$. It will be noted that the agreement with Malus's law may be greatly improved, but that the alternative values will not fit the correlation experiments discussed in Sec. IV. We do not know the accuracy with which Malus's law has been tested with single-photon signals and it is already well understood that results of experiments with macroscopic light cannot be extrapolated to single-photon signals. (An empirical test of Malus's law with such signals will be discussed in Sec. V.) It may be also that our simplified model [defined by Eqs. (2.6), (2.7), and (2.10)] does not allow a single choice of values of $\beta$ and $\gamma$ valid for all experiments. This is a problem to be considered in the future.

We must now consider real polarizers. Among the large number of those existing, we shall study only the two kinds which are of interest in our analysis of experments: piles of plates and the calcite Glan-Thompson prism. Real polarizers are characterized by two efficiency parameters $\epsilon_{M}$ and $\epsilon_{m}$ corresponding to the maximum and minimum transmission of a polarized beam when the polarizer is rotated.

The behavior of a calcite prism can be analyzed with reference to Fig. 3. There, we fix the $y$ axis perpendicular to the plane of the figure and the $x$ axis in the plane but perpendicular to the direction of propagation of the ray. For an incoming signal with electric field components $\left(E_{x}, E_{y}\right)$, the $E_{y}$ component is totally reflected in the balsam layer of the prism (represented by a diagonal straight line in Fig. 3), while the $E_{x}$ component is transmitted with an amplitude $\sqrt{\epsilon_{M}} E_{x}$ (the transmitted part of $E_{y}$ is extremely small and may be neglected). Then, according to classical optics, the transmitted signal should have components $\left(\sqrt{\epsilon_{M}} E_{x}, 0\right)$. In stochastic optics, however, there is some noise which goes into the outgoing channel. In the first place, the $E_{2 y}$ component of the noise ray $\mathbf{E}_{2}$

TABLE I. Predicted detection rate with two perfect polarizers in series. The first row shows the value of $R\left(\theta^{\prime}\right)$ with $\beta$ and $\gamma$ as used in the rest of the paper. The last row shows the quantum-optical prediction.

\begin{tabular}{lcccccc}
\hline \multicolumn{1}{c}{$\beta^{2}$} & $\gamma$ & $\theta^{\prime}=0$ & $\pi / 8$ & $\pi / 4$ & $3 \pi / 8$ & $\pi / 2$ \\
\hline 2.204 & 1.430 & 1.000 & 0.864 & 0.585 & 0.253 & 0.079 \\
2.282 & 1.642 & 1.000 & 0.854 & 0.556 & 0.190 & 0.025 \\
2.36 & 1.375 & 1.000 & 0.856 & 0.560 & 0.242 & 0.081 \\
2.56 & 1.275 & 1.000 & 0.857 & 0.557 & 0.252 & 0.099 \\
Malus's law & & 1.000 & 0.854 & 0.500 & 0.146 & 0.000 \\
\hline \hline
\end{tabular}




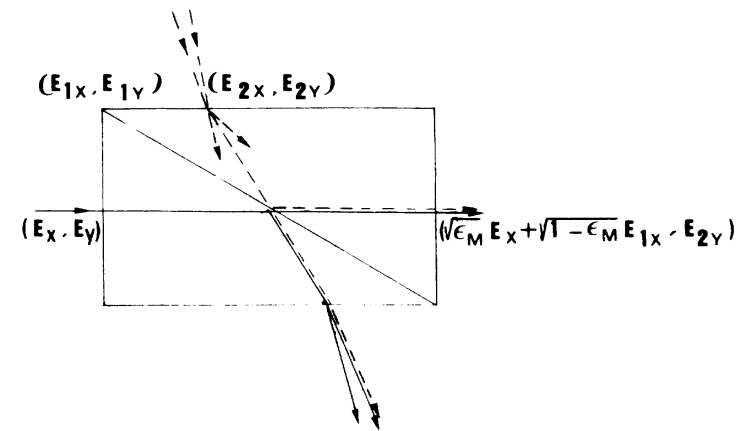

FIG. 3. The action of a Glan-Thompson calcite prism according to stochastic optics. The amplitude interference between the signal (solid line) and the zero-point noise (dashed lines) is important.

is totally reflected and goes into the transmitted channel, but also a small amount of the $E_{1 x}$ component of the noise ray $\mathbf{E}_{1}$ goes there. Then, the transmitted signal has components

$$
\left(\sqrt{\epsilon_{M}} E_{x}+\sqrt{\left(1-\epsilon_{M}\right)} E_{1 x}, E_{2 y}\right) .
$$

The coefficient $\sqrt{\left(1-\epsilon_{M}\right)}$ of $E_{1 x}$ is derived from the following argument. If the amplitude $\sqrt{\epsilon_{M}} E_{x}$ of the $x$ component of the incident signal is transmitted, then an amplitude $\sqrt{\left(1-\epsilon_{M}\right)} E_{x}$ should be reflected by energy conservation (assuming no absorption). However, the incidence of the signal $\mathbf{E}$ on the balsam layer of the prism is the same as that of the noise ray $\mathbf{E}_{1}$, and so the fractions transmitted and reflected should be the same. Hence the intensity of the outgoing signal is just the sum of the square moduli of the components (3.15), i.e.,

$I=\left|\beta \sqrt{\epsilon_{M}} \cos \Phi+\sqrt{\left(1-\epsilon_{M}\right)} \cos \Phi_{1} \exp \left(i \Psi_{1}\right)\right|^{2}+\sin ^{2} \Phi_{2}$.

This agrees with (3.6) for a perfect polarizer with $\epsilon_{M}=1$. From (3.16) we obtain the function $P(\Phi)$ [compare with (3.8)]

$$
\begin{gathered}
P(\Phi)=\xi\left\langle\left(\left|\beta \sqrt{\epsilon_{M}} \cos \Phi+\sqrt{\left(1-\epsilon_{M}\right)} \cos \Phi_{1} \exp \left(i \Psi_{1}\right)\right|^{2}\right.\right. \\
\left.\left.+\sin ^{2} \Phi_{2}-\gamma\right)_{+}\right\rangle_{\Phi_{1}, \Phi_{2}, \Psi_{1}}
\end{gathered}
$$

In order to study the pile of plates polarizer, we must first consider a single plate. If an incident signal has components $\left(E_{x}, E_{y}\right)$ and it arrives at the plate at the Brewster angle, or very close to it (see Fig. 4), then ac-

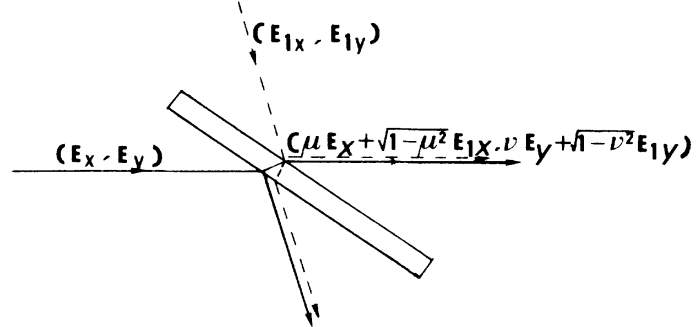

FIG. 4. The action of a single plate polarizer according to stochastic optics.

cording to classical optics, one of the components is fully transmitted, say, $E_{x}$, while the other one is partially reflected and partially transmitted, so that the transmitted signal will have components $\left(\mu E_{x}, v E_{y}\right)$ with

$$
\mu^{2} \simeq 1, \quad v^{2}=2 n^{2} /\left(1+n^{4}\right) \simeq 0.7,
$$

$n$ being the refraction index of the glass plate. In practice, $\mu$ is not exactly unity and so we shall continue using this parameter. If there is no absorption, by conservation of energy the reflected beam will have components $\left(\left(1-\mu^{2}\right)^{1 / 2} E_{x},\left(1-v^{2}\right)^{1 / 2} E_{y}\right)$. In stochastic optics the "relevant part of the noise" which mixes with the signal, as shown in Fig. 4 , has components $\left(E_{1 x}, E_{1 y}\right)$ in the incoming channel. As a result, the transmitted signal plus noise has components

$$
\left(E_{x}^{1}, E_{y}^{1}\right)=\left(\mu E_{x}+\left(1-\mu^{2}\right)^{1 / 2} E_{1 x}, v E_{y}+\left(1-v^{2}\right)^{1 / 2} E_{1 y}\right) .
$$

If there are $N$ plates in the polarizer, we must perform the transformation $N$ times, with the result

$$
\left[\sqrt{\epsilon_{M}} E_{x}+\sqrt{\left(1-\epsilon_{M}\right)} E_{0 x}, \sqrt{\epsilon_{m}} E_{y}+\sqrt{\left(1-\epsilon_{m}\right)} E_{0 y}\right] .
$$

This expression looks like (3.15), but now $E_{0 x}$ and $E_{0 y}$ are different random variables from before whose probability distribution should be found. It is obvious that $E_{0 x}$ and $E_{0 y}$ are complex variables with zero mean, and it is reasonable to assume that the mean intensity is $\frac{1}{2}$. However, the exact probability distribution depends on specific assumptions; for instance, whether noise inputs at different plates are correlated or not. We shall not study this problem in detail in the present paper, and we shall assume that $E_{0 x}$ and $E_{0 y}$ are similar to a single noise intervention. This gives

$$
\begin{aligned}
P(\Phi)=\xi & <\left[\left|\beta \sqrt{\epsilon_{M}} \cos \Phi+\sqrt{\left(1-\epsilon_{M}\right)} \cos \Phi_{0} \exp \left(i \Psi_{0}\right)\right|^{2}\right. \\
& \left.\left.+\left|\beta \sqrt{\epsilon_{M}} \sin \Phi+\sqrt{\left(1-\epsilon_{m}\right)} \sin \Phi_{0} \exp \left(i \chi_{0}\right)\right|^{2}-\gamma\right]_{+}\right\rangle_{\Phi_{0}, \Psi_{0}, \chi_{0}} .
\end{aligned}
$$

The differences with (3.17) are, first, that $\epsilon_{m}$ is not zero, and second, that the $x$ and the $y$ components of the zeropoint intervention are now correlated because they come from the same noise ray. [The correlation is manifest in the use of $\cos \Phi_{0}$ and $\sin \Phi_{0}$ instead of $\cos \Phi_{1}$, and $\sin \Phi_{2}$ as in (3.17)]. In Sec. IV, we shall use (3.21) for piles of plates and similar polarizers for instance, the prism used in the second experiment of Aspect, Grangier, and Roger. ${ }^{5}$ 


\section{OPTICAL TESTS OF THE BELL INEQUALITIES}

The most obviously nonclassical predictions of quantum mechanics are related to pairs of correlated particles (or, more generally, signals). For instance, the two "photons" emitted in an atomic cascade (see Fig. 5) have a correlation in polarization which seems impossible to understand classically. Bell proposed that the probability of a coincidence count should be calculated by an expression which, with some modification, can be written

$p_{12}(a, b)=\int \rho_{12}\left(\lambda_{1}, \lambda_{2}\right) P_{1}\left(\lambda_{1}, a\right) P_{2}\left(\lambda_{2}, b\right) d \lambda_{1} d \lambda_{2}$.

Here $\lambda_{1}\left(\lambda_{2}\right)$ represents the set of auxiliary variables specifying the state of the left (right) "photon," these variables having a probability density $\rho_{12}\left(\lambda_{1}, \lambda_{2}\right)$. $P_{1}\left(\lambda_{1}, a\right)$ is the probability that a photon with auxiliary variables $\lambda_{1}$ crosses a polarizer at angle $a$ and similarly for $P_{2}\left(\lambda_{2}, b\right)$. (In most papers on the subject a single label $\lambda$ is used for all the variables that, for later convenience, we represent here by $\lambda_{1}, \lambda_{2}$.) These functions must fulfill the obvious requirements

$$
\rho_{12} \geq 0, \quad 0 \leq P_{1}, P_{2} \leq 1 .
$$

The condition of locality is contained in formula (4.1) because neither $P_{1}$ depends on $b$ nor $P_{2}$ on $a$, nor $\rho_{12}$ on either $a$ or $b$. Then quantum optics predicts, for the case of perfect polarizers and detectors,

$$
p_{12}(a, b)=\frac{1}{2} \cos ^{2}(a-b) \text {. }
$$

Bell showed that such a value of $p_{12}$ is not compatible with (4.1) and (4.2). The problem is that neither perfect polarizers nor perfect detectors exist.

Now we study the predictions of stochastic optics for correlated-"photon"-pair experiments. First we consider perfect polarizers. Stochastic optical predictions are consistent with the general local realist expression (4.1), with $\lambda_{1}\left(\lambda_{2}\right)$ being the polarization parameters $\phi_{1}, \Psi_{1}$, and $X_{1}$ $\left(\phi_{2}, \Psi_{2}, X_{2}\right)$ of an atomic signal, $a(b)$ the setting of the left (right) polarizer, and $P_{1}\left(P_{2}\right)$ the function given in (3.8) with $\theta=a,(\theta=b)$. It remains only to specify a suitable value for the density factor $\rho_{12}\left(\lambda_{1}, \lambda_{2}\right)$ appearing in (4.1). We have not yet attempted to formulate a detailed model of the emission process in stochastic optics. Within the theory, as so far developed, there is only one obvious requirement, namely, that our description of photon pairs should be consistent with our description of single photons, that is,

$$
\begin{aligned}
d \lambda_{1} \int \rho_{12}\left(\lambda_{1}, \lambda_{2}\right) d \lambda_{2} & =\rho\left(\lambda_{1}\right) d \lambda_{1} \\
& =\left(4 \pi^{2}\right)^{-1} \sin \left(2 \phi_{1}\right) d \phi_{1} d \Psi_{1} d \chi_{1}
\end{aligned}
$$

The procedure we have adopted is to put, for the $0-1-0$ cascade,

$$
\rho_{12}\left(\lambda_{1}, \lambda_{2}\right)=(2 \pi)^{-1} \rho\left(\lambda_{1}\right) \delta\left(\phi_{1}-\phi_{2}\right) \delta\left(\Psi_{1}-\Psi_{2}\right),
$$

and to use the same expression for the 1-1-0 cascade, except that $\delta\left(\phi_{1}-\phi_{2}\right)$ is replaced by $\delta\left(\pi / 2-\phi_{1}-\phi_{2}\right)$. Taken literally, this implies a perfect correlation between $\phi_{1}$ $\left(\Psi_{1}\right)$ and $\phi_{2}\left(\Psi_{2}\right)$, with statistical independence for $\chi_{1}$ and $\chi_{2}$. However, we stress that we have chosen this particular joint distribution solely for computational simplicity. We do not believe that such perfect correlation is a necessary feature either in stochastic optics or in hiddenvariable theories, in general.

In the ideal case Eq. (4.1) becomes

$$
\begin{aligned}
p_{12}(a, b) & =\left\langle P\left(\Phi_{a}\right) P\left(\Phi_{b}\right)\right\rangle_{\lambda_{1} \lambda_{2}} \\
& =\left(4 \pi^{2}\right)^{-1} \int \sin (2 \phi) d \phi d \Psi d \chi P\left(\Phi_{a}\right) P\left(\Phi_{b}\right),
\end{aligned}
$$

where we have removed the lower indices of $\phi_{1}, \Psi_{1}$, and $\chi_{1}$, and we define

$\cos \left(2 \Phi_{a}\right)=\cos (2 \phi) \cos (2 a)+\sin (2 \phi) \sin (2 a) \cos \Psi$,

with a similar expression for $\Phi_{b}$. The integral (4.6) is straightforward, but it is illustrative to solve it using the expansion (3.9), which leads to

$$
\begin{aligned}
& p_{12}(a, b) \\
& =\xi^{2}\left(\beta^{2}-\gamma\right)^{2}\left(a_{0}^{2}+\frac{1}{3} a_{1}^{2} \cos [2(a-b)]\right. \\
& \left.+\frac{1}{20} a_{2}^{2}\{1+3 \cos [4(a-b)]\}+\cdots\right) .
\end{aligned}
$$

A comparison with the quantum optical prediction (4.3), taking the relation (2.10) between $\xi$ and $\eta$ into account, shows that quantum optics corresponds to approximating the function $P(\Phi)$ as in (3.10). However, stochastic optics predicts a definite deviation from quantum optics due to the presence of higher Legendre polynomials in the expansion of $P(\Phi)$. The size of this deviation depends on the specific model that we use [Eqs. (2.6), (2.7), and (2.10)], but an order of magnitude estimate can be obtained from (4.8) and (3.12). Putting $a-b=\theta$, we get

$$
\delta p / \bar{p}=\left(2 / \pi \int_{0}^{\pi / 2}\left[p_{q}(\theta)-p_{s}(\theta)\right]^{2} d \theta\right]^{1 / 2} /\left[2 / \pi \int_{0}^{\pi / 2} p(\theta) d \theta\right] \simeq 0.06
$$

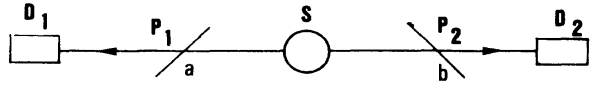

FIG. 5. Diagram of a typical atomic-cascade experiment to test the homogeneous Bell inequalities. where $p_{q}\left(p_{s}\right)$ is the quantum (stochastic) optical prediction. In those experiments, where the coincidence rate has been measured for many values of $\theta$, the errors are of the order of (4.9) or greater. It is interesting that the most accurate measurements have involved the angles 
$\theta=\pi / 8$ and $\theta=3 \pi / 8$, where the predictions of stochastic and quantum optics are quite close to each other. A more detailed comparison follows.

The quantum-optical prediction for the coincidence rate in an experiment with the arrangement of Fig. 5, using a $0-1-0$ or 1-1-0 cascade or the decay of the metastable state of atomic hydrogen, is, for real experiments, ${ }^{2}$

$$
R_{12}(a, b)_{\mathrm{q}}=\frac{1}{4} \eta_{1} \eta_{2} f_{12} R_{0}\left[\epsilon_{+}^{1} \epsilon_{+}^{2} \pm F \epsilon_{-}^{1} \epsilon_{-}^{2} \cos 2(a-b)\right]
$$

(plus sign for 0-1-0 and hydrogen decay; minus sign for 1-1-0), where $a$ and $b$ are the two polarizer settings. Here $\eta_{i}(i=1,2)$ represents the quantum efficiency of detector $i$ and

$$
\epsilon_{+}^{i}=\epsilon_{M}^{i}+\epsilon_{m}^{i}, \quad \epsilon_{-}^{i}=\epsilon_{M}^{i}-\epsilon_{m}^{i},
$$

where $\epsilon_{M}^{i}$ and $\epsilon_{m}^{i}$ represent the maximum and minimum probabilities for a linearly polarized photon to pass through polarizer $i$ (see Sec. III). $f_{12}$ is the joint probability that the two photons of a cascade enter into the appropriate lens collecting systems, while $F$ is a factor, slightly smaller than unity, which corrects for the depolarizing effect of the lens systems. $R_{0}$ is the rate at which cascades occur.

The corresponding prediction for the rate with the polarizers removed is

$$
R_{12}(\infty, \infty)=\eta_{1} \eta_{2} f_{12} R_{0} .
$$

Most of the experiments designed to test the Bell inequalities using single-channel polarizers have measured the quantity

$$
r_{12}(\theta)=\frac{R_{12}(0, \theta)}{R_{12}(\infty, \infty)},
$$

for which the quantum-optical prediction is

$$
r_{12}(\theta)_{\mathrm{q}}=\frac{1}{4}\left[\epsilon_{+}^{1} \epsilon_{+}^{2} \pm F \epsilon_{-}^{1} \epsilon_{-}^{2} \cos (2 \theta)\right] \text {. }
$$

The Bell inequality tested in these experiments states that the Freedman parameter

$$
\delta=\left|r_{12}(\pi / 8)-r_{12}(3 \pi / 8)\right|
$$

satisfies

$$
\delta \leq \frac{1}{4} .
$$

The expression (4.14) gives

$$
\delta_{q}=\left(2^{1 / 2} / 4\right) F \epsilon_{-}^{1} \epsilon_{-}^{2},
$$

and it follows that, with experimental conditions in which $\epsilon_{-}^{i}$ and $F$ are reasonably close to 1 , quantum optics predicts a violation of (4.16).

A total of eight optical experiments ${ }^{4-8,17-20}$ have been performed to test (4.14) and (4.16) (or similar inequalities). Broadly speaking, seven experiments have confirmed, within experimental error, the prediction (4.14) for a range of angles, and in particular they have demonstrated that a Bell inequality is violated. The eighth found, within experimental error, that (4.17) was refuted and (4.16) satisfied. The received wisdom is that there is a systematic error of some kind in this experiment. One of our main aims in this section is to demonstrate that this view may well be mistaken; we shall show that the model of stochastic optics predicts values of $\delta$ which come reasonably close (within two standard deviations) to the experimental values in all the experiments. Naturally, this means that, for the seven experiments which confirm quantum optics, stochastic optics gives values of $r_{12}(\theta)$ reasonably close to its quantum value (4.14). But stochastic optics predicts that there is an important difference between the calcite polarizers used by Holt and Pipkin ${ }^{20}$ and those used in the other seven experiments. This implies that $(4.14)$ is approximately valid for pile-of-plates polarizers but not at all valid for calcite polarizers. We note that the second experiment of Aspect, Grangier, and Roger ${ }^{5}$ used polarizing cubes rather than piles of plates. Nevertheless, we consider it more appropriate to consider these cubes as a close relative of the pile-of-plates family, and therefore have used (3.21) rather than (3.17) to model their action on the incident signals. The 17 different layers of dielectric coating in the middle of the cube may plausibly be considered to give a net effect resembling a pile of plates.

Our expression for $R_{12}(\infty, \infty)$ is then

$$
R_{12}(\infty, \infty)=\xi_{1} \xi_{2} g_{12} R_{0}\left(\beta^{2}-\gamma\right)^{2},
$$

where $g_{12}$ is the joint probability that the two cascade signals enter their respective lens systems. It will be recalled that, in stochastic optics, the signals are considered to be narrow needles of radiation, so this is a valid expression in the approximation where we neglect the very small number of needles which hit the edge of the collecting lens. Note that $g_{12}$ could be the same as $f_{12}$ in (4.12), but that since it actually cancels in subsequent calculations it is not necessary that this be so. The corresponding expression for $R_{12}(a, b)$ is

$$
R_{12}(a, b)=g_{12} R_{0}\left\langle P_{1}\left(\Phi_{a}\right) P_{2}\left(\Phi_{b}\right)\right\rangle_{\lambda_{1}, \lambda_{2}},
$$

where $P_{i}(\Phi)$ is given by (2.10), that is, for example,

$$
P_{1}(\Phi)=\xi_{1}\left\langle\left[I_{1}(\Phi)-\gamma\right]_{+}\right\rangle_{\Phi_{1}, \Phi_{2}}
$$

where the "intensity" $I(\Phi)$ must be computed using the expression (3.17) or (3.21) as appropriate, and this is why our model gives a substantial difference between the two types of polarizer.

Now the quantity $r_{12}(\theta)$ is given by

$r_{12}(\theta)=\xi_{1}^{-1} \xi_{2}^{-1}\left(\beta^{2}-\gamma\right)^{-2}\left\langle P_{1}\left(\Phi_{a}\right) P_{2}\left(\Phi_{b}\right)\right\rangle_{\lambda_{1}, \lambda_{2}}$,

where, as in (4.7)

$$
\Phi_{a}=\Phi(a, \lambda), \quad \lambda=(\phi, \Psi, \chi), \quad a-b=\theta .
$$

It is now a straightforward matter to compute $r_{12}(\theta)$ by substituting (3.17) or (3.21), as appropriate, together with (4.6) into (4.21).

The quantum-mechanical prediction (4.17) for the Freedman parameter contains a factor $F$, which takes into account the depolarizing effect on the emitted photons by the lens systems. This factor would not appear (i.e., $F=1$ ) if the two photons, whose correlations in po- 
larization are measured, were emitted in exactly opposite directions and arrived at the polarizers without any change in direction. Actually, the atomic cascade being a "three-body" decay, the two photons can be emitted in any two arbitrary directions, collected within some solid angle, and change direction in the lenses before arriving at the polarizers. All this produces a decrease in the polarization correlation whose calculation according to quantum optics is straightforward, although cumbersome. The calculation has no specifically quantal feature and could be incorporated straightforwardly also in stochastic optics, producing a less strong correlation than (4.21). As the effect is not very large (less than $2 \%$ in five of the experiments), but implies additional assumptions and a lengthy calculation, we prefer simply to multiply the stochastic optics prediction for (4.15) by the factor $F$; that is, to follow the same procedure as used in the quantum-optics calculation.

The parameter $S$ measured in the second experiment of Aspect, Grangier, and Roger ${ }^{5}$ was

$$
\begin{aligned}
S= & E(0, \pi / 8)+E(\pi / 4, \pi / 8)+E(\pi / 4,3 \pi / 8) \\
& -E(0,3 \pi / 8),
\end{aligned}
$$

where

$$
E(a, b)=\frac{R_{++}(a, b)+R_{--}(a, b)-R_{+-}(a, b)-R_{-+}(a, b)}{R_{++}(a, b)+R_{--}(a, b)+R_{+-}(a, b)+R_{-+}(a, b)} .
$$

In this experiment two-channel polarizers were used so, instead of the single quantity $R_{12}$, the four quantities $R_{++}, R_{+-}, R_{-+}, R_{--}$were measured for various polarizer orientations. The polarizers used were symmetrical between the two channels, so that, within experimental error,

$$
R_{++}(a, b)=R_{--}(a, b), \quad R_{+-}(a, b)=R_{-+}(a, b) .
$$

Although Aspect et al. reported some unexplained lack of rotational symmetry, the theoretical model they used (that is, quantum optics) was rotationally symmetric, as is stochastic optics. In such models, one finds that $S$ reduces to

$$
S=4 \frac{R_{++}(\pi / 8)-R_{++}(3 \pi / 8)}{R_{++}(\pi / 8)+R_{++}(3 \pi / 8)},
$$

so the prediction for this quantity is obtained from the predicted coincidence rate in a single-channel experiment using either of the two symmetric channels $(+$ or -$)$. It is then a straightforward matter to use the computational procedure outlined above to calculate $S$. Again we have taken account of the depolarizing effect by simply multiplying the perfect-correlation result by $F$ (in this case $F=0.984$ ).

We should point out that, although Aspect et al. ${ }^{5}$ considered "highly reasonable" their auxiliary hypothesis that "the ensemble of actually detected pairs is a faithful sample of all emitted pairs," such a hypothesis is rather meaningless in any model, like ours, in which detection probability is a function of signal intensity. They prove that local realistic theories satisfying this hypothesis must give $|S|$ less than 2 , but reference to their detailed argument shows that their hypothesis implies the property

$$
P_{+}(\theta, \lambda)+P_{-}(\theta, \lambda)=\text { const . }
$$

Our model does not have this property and therefore no contradiction arises from the fact that it gives $S$ greater than 2 .

The parameter tested in the Aspect, Dalibard, and Roger experiment ${ }^{6}$ was

$$
S^{\prime}=\frac{R_{12}(0, \pi / 8)+R_{12}(\pi / 4, \pi / 8)+R_{12}(\pi / 4,3 \pi / 8)-R_{12}(0,3 \pi / 8)-R_{12}(\infty, 3 \pi / 8)-R_{12}(\pi / 4, \infty)}{R_{12}(\infty, \infty)}
$$

In rotationally symmetric models this simplifies to

$$
S^{\prime}=\frac{3 R_{12}(\pi / 8)-R_{12}(3 \pi / 8)-R_{12}(\infty, 3 \pi / 8)-R_{12}(\pi / 4, \infty)}{R_{12}(\infty, \infty)},
$$

and one can show that local realistic theories satisfying certain supplementary assumptions must give $S^{\prime}$ less than zero. Again our theory, since it does not satisfy these assumptions, can give $S^{\prime}$ greater than zero, and indeed it agrees very well with the experimental value of $0.101 \pm 0.020$.
We have computed the values of the tested parameters in the eight experiments. The results are given in Table II. It will be seen that our model explains not only the violation of a homogeneous Bell inequality in seven experiments, but also the violation of $(4.17)$ in the HoltPipkin experiment ${ }^{20}$ (see Fig. 6). Given the crudity of our 
TABLE II. Predictions for the tested parameters in Bell inequality experiments. Values of the tested parameters, as predicted by $(A)$ stochastic optics and $(B)$ quantum optics. Experimental values are given in column $(C)$. The Freedman parameter, Eq. (4.15), was tested in the first six experiments quoted; the parameter $S$, given by Eq. (4.22) in Aspect et al. II; and the parameter $S^{\prime}$, given by Eq. (4.27), in Aspect et al. III. In the Clauser experiment no information was given enabling an estimate of the errors in columns $(A)$ and $(B)$ to be made.

\begin{tabular}{|c|c|c|c|c|}
\hline & $\begin{array}{c}(A) \\
\text { Stochastic Optics }\end{array}$ & $\begin{array}{c}(B) \\
\text { Quantum Optics }\end{array}$ & $\begin{array}{c}(C) \\
\text { Experiment }\end{array}$ & $(A)-(C)$ \\
\hline Freedman-Clauser $^{a}$ & $0.309 \pm 0.007$ & $0.301 \pm 0.007$ & $0.300 \pm 0.008$ & $0.009 \pm 0.010$ \\
\hline Holt-Pipkin ${ }^{b}$ & $0.242 \pm 0.001$ & $0.266 \pm 0.001$ & $0.216 \pm 0.013$ & $0.026 \pm 0.013$ \\
\hline Clauser $^{\mathrm{c}}$ & 0.280 & 0.284 & $0.288 \pm 0.009$ & $-0.008 \pm 0.009$ \\
\hline Fry-Thompson ${ }^{\mathrm{d}}$ & $0.296 \pm 0.007$ & $0.294 \pm 0.007$ & $0.296 \pm 0.014$ & $0.000 \pm 0.016$ \\
\hline Aspect et al. $\mathrm{I}^{\mathrm{e}}$ & $0.313 \pm 0.004$ & $0.308 \pm 0.002$ & $0.307 \pm 0.004$ & $0.006 \pm 0.006$ \\
\hline Perrie et al. ${ }^{\mathrm{f}}$ & $0.260 \pm 0.008$ & $0.272 \pm 0.008$ & $0.268 \pm 0.010$ & $-0.008 \pm 0.013$ \\
\hline Aspect et al. $\mathrm{II}^{\mathrm{g}}$ & $2.715 \pm 0.040$ & $2.70 \pm 0.05$ & $2.694 \pm 0.015$ & $0.018 \pm 0.043$ \\
\hline Aspect et al. III $^{\mathrm{h}}$ & $0.101 \pm 0.010$ & $0.112 \pm 0.010$ & $0.101 \pm 0.020$ & $0.000 \pm 0.022$ \\
\hline
\end{tabular}

${ }^{\mathrm{a}}$ Reference 17.

${ }^{\mathrm{b}}$ Reference 20.

${ }^{\mathrm{C}}$ Reference 18.

${ }^{\mathrm{d}}$ Reference 19.
${ }^{\mathrm{e}}$ Reference 4.

${ }^{\mathrm{f}}$ Reference 8.

${ }^{g}$ Reference 5.

${ }^{\text {h}}$ Reference 6. model, we think that the agreement between its predictions and the results of all eight experiments is satisfactory. At the very least, we would hope that statements in the literature that "local realism" has been experimentally refuted will now cease.

\section{EXPERIMENTS WITH ADDITIONAL POLARIZERS}

The basic cascade experiments of Fig. 5 have been embellished by adding various extra polarizing devices: (i)

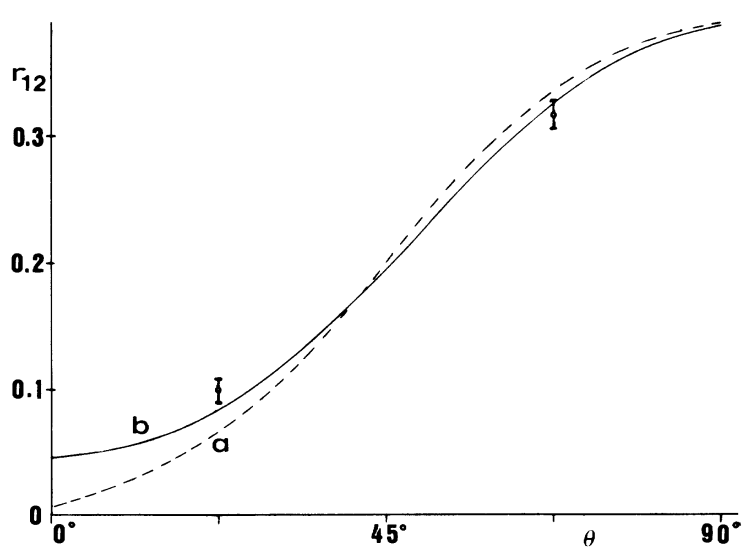

FIG. 6. The predicted coincidence curve $r_{12}(\theta)$ for calcite polarizers according to $a$ quantum optics, and $b$ stochastic optics. The values taken for the efficiencies are those measured by Holt and Pipkin in their experiment with calcite polarizers. The two experimental points obtained by Holt and Pipkin are also displayed.
Clauser $^{21}$ and the Duncan and et al. ${ }^{22}$ placed $\lambda / 4$ wave plates between the source and both of the polarizers; (ii) Duncan et al. ${ }^{22}$ placed a $\lambda / 2$ wave plate between one of the polarizers and its detector; and (iii) Duncan et al. ${ }^{22}$ placed an additional polarizer between one of the polarizers and its detector.

The statistical error bars in all of these experiments are much higher than in the ones discussed in Sec. IV. We have therefore considered it appropriate to simplify our analysis of them by considering all the polarizing devices, that is, linear polarizers and $\lambda / n$ wave plates, to be ideal. We shall find that the discrepancies between stochastic and quantum optics, for all these new types of experiment, in the ideal case, are less than the experimental errors. Although further analysis, along the lines of Sec. IV, with account taken of the actual efficiencies, is desirable, we think this evidence already strengthens our claim that the explanatory power of stochastic optics is greater than that of quantum optics for the whole family of experiments, in this section and Sec. IV.

We consider first the quantum analysis. It is convenient to reverse the historical order, so we take experiment (iii) first. Let the state of the emitted photon pair be denoted

$$
|\Psi\rangle=2^{-1 / 2}\left(|H\rangle_{1}|H\rangle_{2}+|V\rangle_{1}|V\rangle_{2}\right),
$$

where $H$ and $V$ denote horizontal and vertical polarizations. Let the setting of the first polarizer on the righthand side be taken as $H$, with the other two polarizers set at angles $\theta_{1}$, and $\theta_{2}$ to it. Then the passage of the pair of photons through the polarizers is represented by the sequence of collapses 


$$
|\Psi\rangle \rightarrow 2^{-1 / 2}|H\rangle_{1}|H\rangle_{2} \rightarrow 2^{-1 / 2} \cos \theta_{1}\left|\theta_{1}\right\rangle_{1}\left|H_{2}\right\rangle \rightarrow 2^{-1 / 2} \cos \theta_{1} \cos \theta_{2}\left|\theta_{1}\right\rangle_{1}\left|\theta_{2}\right\rangle_{2}
$$

Of course, the order in which the first two collapses occur makes no difference to the end result. Then, from the norm of the last state vector, we obtain the prediction for the coincidence rate

$$
\frac{R_{12}\left(H, \theta_{1}, \theta_{2}\right)}{R_{12}(\infty, \infty, \infty)}=\frac{1}{2} \cos ^{2} \theta_{1} \cos ^{2} \theta_{2} \text {. }
$$

Now we consider experiment (ii) in which the third polarizer is replaced by a $\lambda / 2$ wave plate with retardation $\xi_{2}$ $\left(\xi_{2} \simeq 180^{\circ}\right)$, whose fast axis is set at $\theta_{2}$. The sequence of collapses in this case is

$$
|\Psi\rangle \rightarrow 2^{-1 / 2}|H\rangle_{1}|H\rangle_{2} \rightarrow 2^{-1 / 2} \cos \theta_{1}\left|\theta_{1}\right\rangle_{1}|H\rangle_{2} \rightarrow 2^{-1 / 2} \cos \theta_{1}\left(\cos \theta_{2}\left|\theta_{1}\right\rangle_{1}|F\rangle_{2}+e^{i \xi_{2}} \sin \theta_{2}\left|\Phi_{1}\right\rangle_{1}|S\rangle_{2}\right),
$$

where $F$ and $S$ denote polarizations along the fast and slow axes. In this case, the predicted coincidence rate is

$$
\frac{R_{12}\left(H, \theta_{1}, \theta_{2}, \xi_{2}\right)}{R_{12}(\infty, \infty, \infty)}=\frac{1}{2} \cos ^{2} \theta_{1} .
$$

In experiment (i) we suppose that the $\lambda / 4$ wave plates have their fast axes in the $\mathbf{H}$ direction, and that the two polarizers make angles $\theta_{1}$ and $\theta_{2}$ with these axes. We further suppose the retardations in the two $\lambda / 4$ wave plates to be $\xi_{1}$ and $\xi_{2}$ (both $\left.\simeq 90^{\circ}\right)$. The action on $|\Psi\rangle$ of these two wave plates is

$$
|\Psi\rangle \rightarrow 2^{-1 / 2}\left[|H\rangle_{1}|H\rangle_{2}+e^{i\left(\xi_{1}-\xi_{2}\right)}|V\rangle_{1}|V\rangle_{2}\right]=\left|\Psi^{\prime}\right\rangle,
$$

and the action of the two polarizers is

$$
\begin{aligned}
& \left|\Psi^{\prime}\right\rangle \rightarrow 2^{-1 / 2}\left(\cos \theta_{1} \cos \theta_{2}\right. \\
& \left.\quad+e^{i\left(\xi_{1}-\xi_{2}\right)} \sin \theta_{1} \sin \theta_{2}\right)\left|\theta_{1}\right\rangle_{1}\left|\theta_{2}\right\rangle_{2} .
\end{aligned}
$$

Then the quantum prediction for the coincidence rate is

$$
\begin{aligned}
& \frac{R_{12}\left(\theta_{1}, \theta_{2}, \xi_{1}, \xi_{2}\right)}{R_{12}(\infty, \infty, \infty, \infty)} \\
& =\frac{1}{4}\left[1+\cos \left(2 \theta_{1}\right) \cos \left(2 \theta_{2}\right)\right. \\
& \left.\quad+\sin \left(2 \theta_{1}\right) \sin \left(2 \theta_{2}\right) \cos \left(\xi_{1}-\xi_{2}\right)\right] .
\end{aligned}
$$

We note that this differs from the expression given by Clauser, agreeing only if $\xi_{1}=\xi_{2}$.

The stochastic optical analysis of these experiments now follows. When a $\lambda / n$ plate is inserted between a polarizer and the detector, as in (ii), the counting rate does not change at all. This is because the plate is a type-I device (see Sec. I) which, therefore, does not modify the intensity of any signal if the plate behaves ideally. In consequence, stochastic optics, as well as quantum optics, predicts no change in the correlation. So, the results of the experiment (ii) are easily explained.

The predictions for the other two types of experiment need some calculation. As the statistical errors (of the order of 5-10\%) are higher than in the experiments discussed in Sec. IV, we shall substantially simplify the calculation by making an approximation that also helps in the understanding of the underlying physics. We approximate the detection probability (3.8), for a signal with po- larization parameters $(\phi, \Psi)$ crossing a polarizer at angle $\theta$, by a first-degree polynomial, that is, we use (3.10)

$$
\begin{aligned}
P(x) & =\left\langle\left[\frac{1}{2} \beta^{2}(1+x)+\sin ^{2} \Phi_{0}-\gamma\right]_{+}\right\rangle_{\Phi_{0}} \\
& \simeq\left(\beta^{2}-\gamma\right)\left(\frac{1}{2}+\sqrt{3} / 2 x\right)
\end{aligned}
$$

where $x$ is given by (3.7), that is,

$$
x=\cos (2 \Phi)=\cos (2 \phi) \cos (2 \theta)+\sin (2 \phi) \sin (2 \theta) \cos \Psi .
$$

At first sight it may seem that this approximation of (3.8) is too drastic, but as explained in Sec. IV it introduces errors of only about $6 \%$ in the coincidence rate with two polarizers.

Now, it is easy to calculate the effect of two plates inserted between the source and the two polarizers. A signal emitted by the source with parameters $(\phi, \Psi)$ has electric field components given by $(2.1)$. When it crosses a $\lambda / n$ plate these change according to

$$
\left(\cos \phi, \sin \phi e^{i \Psi}\right) \rightarrow\left(\cos \phi, \sin \phi e^{i\left(\Psi+\xi_{1}\right)}\right),
$$

where $\xi_{1} \simeq 2 \pi / n$, and we have chosen the axes $x$ and $y$ as the fast and slow axes of the $\lambda / n$ plate. A similar expression is obtained for the $\lambda / n$ plate on the other side. (We assume that both plates are inserted with their axes parallel to each other). Therefore, the coincidence rate [see (4.6) and (4.21)] will now be

$$
\begin{gathered}
r_{12}=\frac{1}{2 \pi} \int_{0}^{2 \pi} d \Psi \int_{0}^{\pi / 2} \sin (2 \phi) d \phi\left[\frac{1}{2}+\frac{\sqrt{3}}{2} x_{1}\right] \\
\times\left(\frac{1}{2}+\frac{\sqrt{3}}{2} x_{2}\right],
\end{gathered}
$$

with

$\chi_{i}=\cos (2 \phi) \cos \left(2 \theta_{i}\right)+\sin (2 \phi) \sin \left(2 \theta_{i}\right) \cos \left(\Psi+\xi_{i}\right)$

$$
(i=1,2) \text {. }
$$

The integrals are straightforward and give the quantum optical expression (5.8).

The analysis of the three polarizer experiment is a little more involved. We choose the parameters $\phi$ and $\Psi$ of the source signals, so that the changes with the first and second polarizer are 


$$
\begin{aligned}
& \left(\beta \cos \phi, \beta \sin \phi e^{i \Psi}\right) \\
& \quad \rightarrow\left(\beta \cos \phi, \sin \phi_{1} e^{i \Psi_{1}}\right) \\
& \quad \rightarrow\left(\beta \cos \phi \cos \theta_{2}+\sin \phi_{1} \sin \theta_{2} e^{i \Psi_{1}}, \sin \phi_{2} e^{i \Psi_{2}}\right),
\end{aligned}
$$

where $\left(\phi_{1}, \Psi_{1}\right)$ and $\left(\phi_{2}, \Psi_{2}\right)$ correspond to the two noise interventions. The normalized detection probability of this signal will be, therefore,

$$
\begin{gathered}
Q\left(x_{2}\right)=\left(\beta^{2}-\gamma\right)^{-1}\left\langle\left[\left|\beta \cos \phi \cos \theta_{2}+\sin \phi_{1} \sin \theta_{2} e_{1}^{i \Psi}\right|^{2}\right.\right. \\
\left.\left.+\sin ^{2} \phi_{2}-\gamma\right]_{+}\right\rangle_{\phi_{1}, \phi_{2}, \Psi_{1}} .
\end{gathered}
$$

The approximation (5.9) is still valid, provided that we now define

$$
x_{2}=2\left|\cos \phi \cos \theta_{2}+\sin \phi_{1} \sin \theta_{2} e_{1}^{i \Psi}\right|^{2}-1 .
$$

Then, Eq. (5.11) applies and we obtain, after averaging over $\phi_{1}$ and $\Psi_{1}$,

$$
\begin{aligned}
r\left(\theta_{1}, \theta_{2}\right) & =\left(\frac{1}{2}\right) \cos ^{2} \theta_{1} \cos ^{2} \theta_{2}+\frac{1}{4}\left[1-\sqrt{3}\left(1-\beta^{-2}\right)\right] \sin ^{2} \theta_{2} \\
& \simeq\left(\frac{1}{2}\right) \cos ^{2} \theta_{1} \cos ^{2} \theta_{2}+0.013 \sin ^{2} \theta_{2},
\end{aligned}
$$

which agrees with the quantum-optical prediction (5.3), except for a small term. This expression also agrees with the result of the Stirling experiment by Duncan et al. ${ }^{22}$ within errors.

\section{ACKNOWLEDGMENTS}

We acknowledge the financial support of the Caja de Ahorros de Cantabria. We Also acknowledge the financial support of DGICYT Project No. PB-87-0014 (Spain).
${ }^{1}$ T. W. Marshall and E. Santos, Found Phys. 18, 185 (1988).

${ }^{2}$ J. F. Clauser and A. Shimony, Rep. Prog. Phys. 41, 1881 (1978).

${ }^{3}$ F. Selleri and G. Tarozzi, Riv. Nuovo Cimento 4, 1 (1981).

${ }^{4}$ A. Aspect, P. Grangier, and G. Roger, Phys. Rev. Lett. 47, 460 (1981).

${ }^{5}$ A. Aspect, P. Grangier, and G. Roger, Phys. Rev. Lett. 49, 91 (1982).

${ }^{6}$ A. Aspect, J. Dalibard, and G. Roger, Phys. Rev. Lett. 49, 1804 (1982).

${ }^{7}$ A. Aspect and P. Grangier, Lett. Nuovo Cimento 45, 435 (1985).

${ }^{8}$ W. Perrie, A. J. Duncan, H. J. Beyer, and H. Kleinpoppen, Phys. Rev. Lett. 54, 1790 (1985).

${ }^{9}$ P. Claverie and S. Diner, Int. J. Quantum Chem. 12, Suppl. 1, 41 (1977).

${ }^{10} \mathrm{~T}$. H. Boyer, in Foundations of Radiation Theory and Quantum Electrodynamics, edited by A. O. Barut (Plenum, New York, 1980), p. 49.

${ }^{11}$ L. de la Peña, in Stochastic Processes Applied to Physics and
Other Related Fields, edited by B. Gomez, S. M. Moore, A. M. Rodriguez-Vargas, and A. Rueda (World Scientific, Singapore, 1983), p. 428.

${ }^{12}$ P. L. Knight and L. Allen, Quantum Optics (Pergamon, Oxford, 1983).

${ }^{13}$ E. T. Jaynes, Ref. 10, p. 37.

${ }^{14}$ T. W. Marshall and E. Santos, Phys. Lett. A 107, 164 (1985).

${ }^{15}$ E. Santos, Nuovo Cimento B 22, 201 (1974).

${ }^{16} \mathrm{~T}$. W. Marshall and E. Santos (unpublished).

${ }^{17}$ S. J. Freedman and J. F. Clauser, Phys. Rev. Lett. 28, 938 (1972).

${ }^{18}$ J. F. Clauser, Phys. Rev. Lett. 36, 1223 (1976).

${ }^{19}$ E. S. Fry and R. C. Thompson, Phys. Rev. Lett. 37, 465 (1976).

${ }^{20}$ R. A. Holt and F. M. Pipkin (unpublished). See Refs. 2 and 22.

${ }^{21}$ J. F. Clauser, Nuovo Cimento B 33, 740 (1976).

${ }^{22}$ A. J. Duncan and H. Kleinpoppen, in Quantum Mechanics Versus Local Realism: The Einstein, Podolsky and Rosen Paradox, edited by F. Selleri (Plenum, New York, 1988). 